Math. Model. Nat. Phenom.

Vol. 4, No. 1, 2009, pp. 147-162

DOI: $10.1051 / \mathrm{mmnp} / 20094107$

\title{
Weak Formulations and Solution Multiplicity of Equilibrium Configurations with Coulomb Friction
}

\author{
M. Bostan and P. Hild ${ }^{1}$ \\ Laboratoire de Mathématiques, Université de Franche-Comté \\ CNRS UMR 6623, 16 route de Gray, 25030 Besançon, France
}

\begin{abstract}
This work is concerned with the equilibrium configurations of elastic structures in contact with Coulomb friction. We obtain a variational formulation of this equilibrium problem. Then we propose sufficient conditions for the existence of an infinity of equilibrium configurations with arbitrary small friction coefficients. We illustrate the result in two space dimensions with a simple example.
\end{abstract}

Key words: Coulomb friction, linear elasticity, equilibrium configurations, weak formulations, continuum of solutions.

AMS subject classification: 74G35, 74M10, 74M15.

\section{Introduction and problem set-up}

The equilibrium configurations of structures submitted to contact with Coulomb friction are of current concern in engineering sciences. Many applications can be found in geophysics, robotics, manufacturing processes and more generally in contact mechanics. This study deals with these equilibrium solutions to the dynamic frictional contact problem (and also to the quasi-static problem) in the continuous case. Let us recall that a lot of work has been achieved from a mathematical and/or numerical point of view for the so-called "static Coulomb friction problem" (see,

\footnotetext{
${ }^{1}$ Corresponding author. E-mail: patrick.hild @univ-fcomte.fr
} 
e.g., $[4,6,5,14,20,21]$ ) whose solutions satisfy equilibrium (note that the set of solutions to the static problem is much smaller than the set of equilibrium solutions to the dynamic or quasi-static problem). The main aim of this paper is to discuss on some elementary properties (particularly the variational formulation and some solution multiplicities for small friction coefficients) for the equilibrium problem. The paper is organized as follows.

Next we recall the dynamic, quasi-static, static and equilibrium frictional contact problems. Section 2 is concerned with the determination of the weak formulation of the equilibrium problem which consists in an inequality. In the following section we propose sufficient conditions ensuring, for a given body and given loads, an infinity of equilibrium configurations for arbitrary small friction coefficients. In section 4 we illustrate this result in two space dimensions with some elementary examples which we determine explicitly.

We consider the deformation of an elastic body occupying, in the initial unconstrained configuration a bounded domain $\Omega$ in $\mathbb{R}^{d}, d=2$ or 3 , with Lipschitz boundary, where plane strain assumptions are assumed. The boundary $\partial \Omega=\overline{\Gamma_{D}} \cup \overline{\Gamma_{N}} \cup \overline{\Gamma_{C}}$ of $\Omega$ consists of three non-overlapping domains $\Gamma_{D}, \Gamma_{N}$ and $\Gamma_{C}$ where the superficial measures of $\Gamma_{D}$ and $\Gamma_{C}$ are positive. The body $\Omega$ is submitted to given displacements $\mathbf{U}$ on $\Gamma_{D}$, it is subjected to surface traction forces $\mathbf{F}$ on $\Gamma_{N}$ and the body forces are denoted by $f$. In the initial configuration, the part $\Gamma_{C}$ is considered as the candidate contact surface on a rigid foundation which means that the contact zone cannot enlarge during the deformation process. The contact is assumed to be frictional and the stick, slip and separation zones on $\Gamma_{C}$ are not known in advance. We denote by $\mu \geq 0$ the given friction coefficient on $\Gamma_{C}$.

\subsection{The dynamic problem}

The dynamic unilateral contact problem with the Coulomb friction law consists of finding the displacement field $\mathbf{u}: \Omega \times[0, T] \rightarrow \mathbb{R}^{d}$ satisfying (1.1)-(1.8):

$$
\begin{aligned}
\operatorname{div} \boldsymbol{\sigma}(\mathbf{u})+\mathbf{f} & =\rho \ddot{\mathbf{u}} \quad \text { in } \Omega \times[0, T], \\
\boldsymbol{\sigma}(\mathbf{u}) & =\mathbf{C} \boldsymbol{\varepsilon}(\mathbf{u}) \quad \text { in } \Omega \times[0, T], \\
\mathbf{u} & =\mathbf{U} \quad \text { on } \Gamma_{D} \times[0, T], \\
\boldsymbol{\sigma}(\mathbf{u}) \mathbf{n} & =\mathbf{F} \quad \text { on } \Gamma_{N} \times[0, T],
\end{aligned}
$$

where $\rho>0$ represents the density of the body and the notation $\boldsymbol{\sigma}(\mathbf{u}): \Omega \rightarrow S_{d}$ stands for the stress tensor field lying in $S_{d}$, the space of second order symmetric tensors on $\mathbb{R}^{d}$. The linearized strain tensor field is $\varepsilon(\mathbf{u})=\left(\boldsymbol{\nabla} \mathbf{u}+\nabla^{T} \mathbf{u}\right) / 2$ and $\mathbf{C}$ is the fourth order symmetric and elliptic tensor of linear elasticity.

Afterwards we choose the following notation for any displacement field $\mathbf{v}$ and for any density of surface forces $\boldsymbol{\sigma}(\mathbf{v}) \mathbf{n}$ defined on $\partial \Omega$ (the notation $\mathbf{n}$ represents the unitary outward normal vector on $\partial \Omega$ ):

$$
\begin{gathered}
v_{n}=\mathbf{v} \cdot \mathbf{n}, \quad \mathbf{v}_{T}=\mathbf{v}-v_{n} \mathbf{n} \\
\sigma_{n}(\mathbf{v})=(\boldsymbol{\sigma}(\mathbf{v}) \mathbf{n}) \cdot \mathbf{n}, \quad \boldsymbol{\sigma}_{T}(\mathbf{v})=\boldsymbol{\sigma}(\mathbf{v}) \mathbf{n}-\sigma_{n}(\mathbf{v}) \mathbf{n} .
\end{gathered}
$$


On $\Gamma_{C} \times[0, T]$, the three conditions representing unilateral contact are as follows

$$
u_{n} \leq 0, \quad \sigma_{n}(\mathbf{u}) \leq 0, \quad \sigma_{n}(\mathbf{u}) u_{n}=0
$$

and the Coulomb friction law on $\Gamma_{C} \times[0, T]$ is described by the following conditions (see e.g., [4], p. 21, or [8]):

$$
\left\{\begin{aligned}
\dot{\mathbf{u}}_{T}=\mathbf{0} & \Longrightarrow\left|\boldsymbol{\sigma}_{T}(\mathbf{u})\right| \leq-\mu \sigma_{n}(\mathbf{u}) \\
\dot{\mathbf{u}}_{T} \neq \mathbf{0} & \Longrightarrow \boldsymbol{\sigma}_{T}(\mathbf{u})=\mu \sigma_{n}(\mathbf{u}) \frac{\dot{\mathbf{u}}_{T}}{\left|\dot{\mathbf{u}}_{T}\right|}
\end{aligned}\right.
$$

Finally we prescribe initial conditions for the displacements and the velocities:

$$
\begin{aligned}
& \mathbf{u}_{\mid t=0}=\mathbf{u}^{0}, \\
& \dot{\mathbf{u}}_{\mid t=0}=\mathbf{u}^{1} .
\end{aligned}
$$

The dynamic contact problem (1.1)-(1.8) (with or without friction) remains unsolved concerning the existence and uniqueness of solutions and it shows great mathematical difficulties. A survey of the existing work dealing with close problems to (1.1)-(1.8) and several technical details can be found in [4].

Remark 1. A problem where much interest was focused on in the recent years is the so-called quasi-static problem. It consists of finding a displacement field $\mathbf{u}: \Omega \times[0, T] \rightarrow \mathbb{R}^{d}$ satisfying

$$
\operatorname{div} \boldsymbol{\sigma}(\mathbf{u})+\mathbf{f}=\mathbf{0} \quad \text { in } \Omega \times[0, T]
$$

and conditions (1.2)-(1.7).

The existence of a solution has been proved for the quasi-static problem if the friction coefficient is small enough (see $[1,19])$.

\subsection{The equilibrium problem}

Supposing that $\mathbf{f}, \mathbf{F}, \mathbf{U}$ do not depend on the time but only on the space variable, an equilibrium solution of the dynamic problem is a displacement field which is constant in time, e.g, a field $\mathbf{u}: \Omega \rightarrow \mathbb{R}^{d}$ satisfying (1.9)-(1.14):

$$
\begin{aligned}
\operatorname{div} \boldsymbol{\sigma}(\mathbf{u})+\mathbf{f} & =\mathbf{0} \quad \text { in } \Omega \\
\boldsymbol{\sigma}(\mathbf{u}) & =\mathbf{C} \boldsymbol{\varepsilon}(\mathbf{u}) \quad \text { in } \Omega \\
\mathbf{u} & =\mathbf{U} \quad \text { on } \Gamma_{D} \\
\boldsymbol{\sigma}(\mathbf{u}) \mathbf{n} & =\mathbf{F} \quad \text { on } \Gamma_{N} \\
u_{n} & \leq 0, \quad \sigma_{n}(\mathbf{u}) \leq 0, \quad \sigma_{n}(\mathbf{u}) u_{n}=0 \quad \text { on } \Gamma_{C}, \\
\left|\boldsymbol{\sigma}_{T}(\mathbf{u})\right| & \leq-\mu \sigma_{n}(\mathbf{u}) \quad \text { on } \Gamma_{C} .
\end{aligned}
$$


It is straightforward to check that an equilibrium solution solves both the dynamic and quasi-static problems (in which the initial condition is precisely the equilibrium solution). A study dealing with stability properties of equilibrium configurations in the continuous context can be found in [8].

Remark 2. The so-called static problem consists of finding an equilibrium solution satisfying (1.9)-(1.14) and also (see [4], p. 27):

$$
\left\{\begin{aligned}
\mathbf{u}_{T}=\mathbf{0} & \Longrightarrow\left|\boldsymbol{\sigma}_{T}(\mathbf{u})\right| \leq-\mu \sigma_{n}(\mathbf{u}) \\
\mathbf{u}_{T} \neq \mathbf{0} & \Longrightarrow \boldsymbol{\sigma}_{T}(\mathbf{u})=\mu \sigma_{n}(\mathbf{u}) \frac{\mathbf{u}_{T}}{\left|\mathbf{u}_{T}\right|}
\end{aligned}\right.
$$

This formulation can be seen as an incremental problem in the time discretized quasi-static model. The first existence results were obtained in [16], generalized later, and improved in [3]. These existence results hold for small friction coefficients. In the general case uniqueness does not hold, at least for large friction coefficients (greater than $\sqrt{(1-\nu) / \nu}$, where $0<\nu<1 / 2$ represents Poisson's ratio), see [9, 10]. More recently a first uniqueness result has been obtained in [18] with the assumption that a "regular" solution exists and that the friction coefficient is sufficiently small.

\section{Weak formulation}

Next we give a variational formulation for the equilibrium problem (1.9)-(1.14). We use the forms

$$
a(\mathbf{v}, \mathbf{w})=\int_{\Omega} \boldsymbol{\sigma}(\mathbf{v}): \varepsilon(\mathbf{w}) d \Omega
$$

for any $\mathbf{v}, \mathbf{w} \in H^{1}(\Omega)^{d}$ and

$$
\langle l, \mathbf{v}\rangle=\int_{\Omega} \mathbf{f} \cdot \mathbf{v} d \Omega+\int_{\Gamma_{N}} \mathbf{F} \cdot \mathbf{v} d \Gamma
$$

for any $\mathbf{v} \in H^{1}(\Omega)^{d}$. Finally we introduce the functional

$$
j(\mathbf{v}, \mathbf{w})=-\int_{\Gamma_{C}} \sigma_{n}(\mathbf{v})\left|\mathbf{w}_{T}\right| d \Gamma
$$

which is defined for any $\mathbf{w}$ in $H^{1}(\Omega)^{d}$ but more regularity is required for $\mathbf{v}$. The case where $j(\mathbf{v},$. makes sense involves the space

$$
\tilde{\mathbf{V}}=\left\{\mathbf{v} \in H^{1}(\Omega)^{d}: \operatorname{div} \boldsymbol{\sigma}(\mathbf{v}) \in L^{2}(\Omega)^{d}\right\}
$$

If $\mathbf{v} \in \tilde{\mathbf{V}}$ then $\boldsymbol{\sigma}(\mathbf{v})$ belongs to $H(\operatorname{div}, \Omega)$ and $\sigma_{n}(\mathbf{v})$ is an element of $H^{-\frac{1}{2}}(\partial \Omega)$ (i.e. the dual of $H^{\frac{1}{2}}(\partial \Omega)$ here). Since $\left.H^{-\frac{1}{2}}(\partial \Omega)\right|_{\Gamma_{C}}$ is different from $H^{-\frac{1}{2}}\left(\Gamma_{C}\right)$ we have to suppose in addition that 
$\sigma_{n}(\mathbf{v}) \in H^{-\frac{1}{2}}\left(\Gamma_{C}\right)$. With this assumption, (2.1) makes sense if we replace the integral term by the duality product. For a more precise formulation involving the convenient Sobolev spaces and the set of nonnegative Radon measures, a detailed study can be found in [15]. To avoid more notation, we will skip over the regularity aspects of the functions defined on $\Gamma_{C}$ which are beyond the scope of this paper and we write afterwards integral terms instead of duality pairings.

We introduce the closed convex set:

$$
\mathbf{K}=\left\{\mathbf{v} \in H^{1}(\Omega)^{d}: \mathbf{v}=\mathbf{U} \text { on } \Gamma_{D}, v_{n} \leq 0 \text { on } \Gamma_{C}\right\} .
$$

Finally we assume that $\mathbf{f} \in L^{2}(\Omega)^{d}$ and $\mathbf{F} \in L^{2}\left(\Gamma_{N}\right)^{d}$. The next result is concerned with the weak formulation of (1.9)-(1.14).

Proposition 3. The displacement field $\mathbf{u}$ is a solution for the equilibrium problem (1.9)-(1.14) if and only if $\mathbf{u}$ satisfies the inequality

$$
\mathbf{u} \in \mathbf{K}: a(\mathbf{u}, \mathbf{v}-\mathbf{u})+\mu j(\mathbf{u}, \mathbf{v}-\mathbf{u}) \geq\langle l, \mathbf{v}-\mathbf{u}\rangle, \forall \mathbf{v} \in \mathbf{K}
$$

Remark 4. Note that the weak formulation of this problem is close to that of the static problem (see Remark 2) where $j(\mathbf{u}, \mathbf{v}-\mathbf{u})$ is replaced with $j(\mathbf{u}, \mathbf{v})-j(\mathbf{u}, \mathbf{u})$.

Proof. Assume that $\mathbf{u} \in H^{1}(\Omega)^{d}$ solves (1.9)-(1.14). Obviously $\mathbf{u} \in \mathbf{K}$ and for any test function $\mathbf{v} \in \mathbf{K}$ one gets after integration by parts

$$
\begin{aligned}
\int_{\Omega} \boldsymbol{\sigma}(\mathbf{u}): \varepsilon(\mathbf{v}-\mathbf{u}) d \Omega= & \int_{\Omega} \mathbf{f} \cdot(\mathbf{v}-\mathbf{u}) d \Omega+\int_{\partial \Omega} \boldsymbol{\sigma}(\mathbf{u}) \mathbf{n} \cdot(\mathbf{v}-\mathbf{u}) d \Gamma \\
= & \int_{\Omega} \mathbf{f} \cdot(\mathbf{v}-\mathbf{u}) d \Omega+\int_{\Gamma_{N}} \mathbf{F} \cdot(\mathbf{v}-\mathbf{u}) d \Gamma \\
& +\int_{\Gamma_{C}} \boldsymbol{\sigma}(\mathbf{u}) \mathbf{n} \cdot(\mathbf{v}-\mathbf{u}) d \Gamma \\
= & \langle l, \mathbf{v}-\mathbf{u}\rangle+\int_{\Gamma_{C}} \sigma_{n}(\mathbf{u})\left(v_{n}-u_{n}\right) d \Gamma \\
& +\int_{\Gamma_{C}} \boldsymbol{\sigma}_{T}(\mathbf{u}) \cdot\left(\mathbf{v}_{T}-\mathbf{u}_{T}\right) d \Gamma .
\end{aligned}
$$

Notice that

$$
\int_{\Gamma_{C}} \sigma_{n}(\mathbf{u})\left(v_{n}-u_{n}\right) d \Gamma=\int_{\Gamma_{C}} \sigma_{n}(\mathbf{u}) v_{n} d \Gamma \geq 0
$$

and also that

$$
\begin{aligned}
\int_{\Gamma_{C}} \boldsymbol{\sigma}_{T}(\mathbf{u}) \cdot\left(\mathbf{v}_{T}-\mathbf{u}_{T}\right) d \Gamma & \geq-\int_{\Gamma_{C}} \boldsymbol{\sigma}_{T}(\mathbf{u})|| \mathbf{v}_{T}-\mathbf{u}_{T} \mid d \Gamma \\
& \geq \mu \int_{\Gamma_{C}} \sigma_{n}(\mathbf{u})\left|\mathbf{v}_{T}-\mathbf{u}_{T}\right| d \Gamma \\
& =-\mu j(\mathbf{u}, \mathbf{v}-\mathbf{u}) .
\end{aligned}
$$


Combining (2.3), (2.4), (2.5) yields

$$
a(\mathbf{u}, \mathbf{v}-\mathbf{u})+\mu j(\mathbf{u}, \mathbf{v}-\mathbf{u}) \geq\langle l, \mathbf{v}-\mathbf{u}\rangle, \forall \mathbf{v} \in \mathbf{K} .
$$

Conversely, assume now that $\mathbf{u}$ is a solution for the variational inequality (2.2). Taking $\mathbf{v}=\mathbf{u} \pm \varphi$ with $\varphi \in C^{1}(\bar{\Omega})^{d}$ satisfying $\varphi=0$ on $\Gamma_{D} \cup \Gamma_{C}$, we obtain $a(\mathbf{u}, \boldsymbol{\varphi})=\langle l, \varphi\rangle$ which implies $\operatorname{div} \boldsymbol{\sigma}(\mathbf{u})+\mathbf{f}=\mathbf{0}$ in $\Omega$ and $\boldsymbol{\sigma}(\mathbf{u}) \mathbf{n}=\mathbf{F}$ on $\Gamma_{N}$. Since $\mathbf{u}$ belongs to $\mathbf{K}$ we have also $\mathbf{u}=\mathbf{U}$ on $\Gamma_{D}$ and $u_{n} \leq 0$ on $\Gamma_{C}$. It remains to prove that $\sigma_{n}(\mathbf{u}) \leq 0, u_{n} \sigma_{n}(\mathbf{u})=0$ and $\left|\boldsymbol{\sigma}_{T}(\mathbf{u})\right| \leq-\mu \sigma_{n}(\mathbf{u})$ on $\Gamma_{C}$. Observe that the variational inequality (2.2) implies for any $\mathbf{v} \in \mathbf{K}$

$$
\int_{\Gamma_{C}} \sigma_{n}(\mathbf{u})\left(v_{n}-u_{n}\right) d \Gamma+\int_{\Gamma_{C}} \boldsymbol{\sigma}_{T}(\mathbf{u}) \cdot\left(\mathbf{v}_{T}-\mathbf{u}_{T}\right) d \Gamma-\mu \int_{\Gamma_{C}} \sigma_{n}(\mathbf{u})\left|\mathbf{v}_{T}-\mathbf{u}_{T}\right| d \Gamma \geq 0 .
$$

Taking $\mathbf{v} \in \mathbf{K}$ such that $\mathbf{v}_{T}=\mathbf{u}_{T}, v_{n}=0$ on $\Gamma_{C}$ we deduce that

$$
\int_{\Gamma_{C}} \sigma_{n}(\mathbf{u}) u_{n} d \Gamma \leq 0
$$

Take now $\mathbf{v} \in \mathbf{K}$ such that $\mathbf{v}_{T}=\mathbf{u}_{T}, v_{n}=2 u_{n}$ on $\Gamma_{C}$ and this time we obtain

$$
\int_{\Gamma_{C}} \sigma_{n}(\mathbf{u}) u_{n} d \Gamma \geq 0
$$

From (2.7), (2.8) we deduce that $\int_{\Gamma_{C}} \sigma_{n}(\mathbf{u}) u_{n} d \Gamma=0$ and therefore (2.6) becomes

$$
\int_{\Gamma_{C}} \sigma_{n}(\mathbf{u}) v_{n} d \Gamma+\int_{\Gamma_{C}}\left\{\boldsymbol{\sigma}_{T}(\mathbf{u}) \cdot\left(\mathbf{v}_{T}-\mathbf{u}_{T}\right)-\mu \sigma_{n}(\mathbf{u})\left|\mathbf{v}_{T}-\mathbf{u}_{T}\right|\right\} d \Gamma \geq 0
$$

for any $\mathbf{v} \in \mathbf{K}$. In particular, by taking $\mathbf{v}$ such that $\mathbf{v}_{T}=\mathbf{u}_{T}$ and $v_{n} \leq 0$ on $\Gamma_{C}$ we have $\int_{\Gamma_{C}} \sigma_{n}(\mathbf{u}) v_{n} d \Gamma \geq 0$ saying that $\sigma_{n}(\mathbf{u}) \leq 0$ on $\Gamma_{C}$. Since we have already proved that

$$
\int_{\Gamma_{C}} \sigma_{n}(\mathbf{u}) u_{n} d \Gamma=0
$$

and $\sigma_{n}(\mathbf{u}) u_{n} \geq 0$ on $\Gamma_{C}$, it follows that $\sigma_{n}(\mathbf{u}) u_{n}=0$ on $\Gamma_{C}$. Consider now test functions $\mathbf{v}=$ $\mathbf{u}+\mathbf{w}$ for any $\mathbf{w}$ satisfying $\mathbf{w}=\mathbf{0}$ on $\Gamma_{D}$ and $w_{n}=0$ on $\Gamma_{C}$. Such a test function belongs to $\mathbf{K}$ and by applying (2.6) we deduce that

$$
-\int_{\Gamma_{C}} \boldsymbol{\sigma}_{T}(\mathbf{u}) \cdot \mathbf{w}_{T} d \Gamma \leq-\mu \int_{\Gamma_{C}} \sigma_{n}(\mathbf{u})\left|\mathbf{w}_{T}\right| d \Gamma .
$$

Take now $\mathbf{w}_{T}(x)=-\boldsymbol{\sigma}_{T}(\mathbf{u})(x) z(x) /\left|\boldsymbol{\sigma}_{T}(\mathbf{u})(x)\right|$ if $x \in \Gamma_{C}, \boldsymbol{\sigma}_{T}(\mathbf{u})(x) \neq \mathbf{0}$ and $\mathbf{w}_{T}(x)=\mathbf{0}$ if $x \in \Gamma_{C}, \boldsymbol{\sigma}_{T}(\mathbf{u})(x)=\mathbf{0}$, with an arbitrary nonnegative function $z$ on $\Gamma_{C}$. We have

$$
-\boldsymbol{\sigma}_{T}(\mathbf{u})(x) \cdot \mathbf{w}_{T}(x)=\left|\boldsymbol{\sigma}_{T}(\mathbf{u})(x)\right| z(x), \quad\left|\mathbf{w}_{T}(x)\right| \leq z(x), \forall x \in \Gamma_{C},
$$

and therefore (2.9) implies

$$
\int_{\Gamma_{C}}\left|\boldsymbol{\sigma}_{T}(\mathbf{u})(x)\right| z(x) d \Gamma \leq-\mu \int_{\Gamma_{C}} \sigma_{n}(\mathbf{u})(x) z(x) d \Gamma,
$$

saying that $\left|\boldsymbol{\sigma}_{T}(\mathbf{u})\right| \leq-\mu \sigma_{n}(\mathbf{u})$ on $\Gamma_{C}$. 
Remark 5. 1. It is easy to check that if $\mathbf{u}$ solves the problem (1.9)-(1.14) for given loads $(\mathbf{f}, \mathbf{F}, \mathbf{U})$ then for any $\lambda \geq 0$, the field $\lambda \mathbf{u}$ solves the problem (1.9)-(1.14) for given loads $(\lambda \mathbf{f}, \lambda \mathbf{F}, \lambda \mathbf{U})$. This property holds also for the static problem.

2. When $\mathbf{f}=\mathbf{F}=\mathbf{U}=\mathbf{0}$, the field $\mathbf{u}=\mathbf{0}$ solves (1.9)-(1.14). In addition there also exist (in some cases) some nontrivial equilibrium solutions which are called "wedged configurations" $($ see $[2,7])$. Such solutions correspond to configurations which remain in a deformed state when the loads are removed. This phenomenon does not occur for the static problem where it is easy to check that $\mathbf{u}=\mathbf{0}$ is the unique solution when the data $(\mathbf{f}, \mathbf{F}, \mathbf{U})$ vanishes.

\section{Sufficient conditions for solution multiplicity to the equilib- rium problem for arbitrary small friction}

The aim of this section is to obtain for a given geometry, material characteristics (incorporated in the elasticity tensor $\mathbf{C}$ ) and loads $(\mathbf{f}, \mathbf{F}, \mathbf{U})$, a sufficient condition for the existence of infinitely many solutions to the equilibrium problem (1.9)-(1.14) for arbitrary small friction coefficients. Of course our result is weaker than the one which claims that infinitely many solutions to the static problem may exist for arbitrary small friction coefficients. Afterwards in order to focus on the dependence on the friction coefficient we denote, for a given geometry, material characteristics and data $(\mathbf{f}, \mathbf{F}, \mathbf{U})$, by $\mathbf{u}_{\mu}$ a solution of (1.9)-(1.14) corresponding to the friction coefficient $\mu$. We begin with some elementary remarks dealing with existence of equilibrium configurations.

Remark 6. 1 . When $\mu=0$, an equilibrium solution $\mathbf{u}_{0}$ exists, it is the unique solution of (1.9)(1.14) and it coincides with the unique solution of the static frictionless unilateral contact problem (according to Stampacchia's theorem).

2. If $\mathbf{u}_{\bar{\mu}}$ is an equilibrium solution of (1.9)-(1.14) then this displacement field $\mathbf{u}_{\bar{\mu}}$ is also a equilibrium solution for any $\mu \geq \bar{\mu}$ (note that this property does not hold for the static problem). Hence $\mathbf{u}_{0}$ is an equilibrium solution for any friction coefficient.

Next we focus on non-uniqueness of the solution to the equilibrium problem.

Proposition 7. Suppose that there exists $\varepsilon>0$ such that for any friction coefficient $\mu \in[0, \varepsilon]$ there exists an equilibrium solution $\mathbf{u}_{\mu}$ to (1.9)-(1.14) satisfying the following property: $\mathbf{u}_{\mu} \neq \mathbf{u}_{\bar{\mu}}$ for any $\mu$ and $\bar{\mu}$ in $[0, \varepsilon]$ such that $\mu \neq \bar{\mu}$.

Then for any positive friction coefficient $\mu$ there exists an infinity of equilibrium configurations. More precisely, any $\mathbf{u}_{\bar{\mu}}$ with $\bar{\mu} \in[0, \min (\mu, \varepsilon)]$ solves (1.9)-(1.14).

Proof. Straightforward according to the previous Remark 62 .

The previous proposition claims that if there exists a one-to-one map between the friction coefficients in $[0, \varepsilon]$ and some solutions to the equilibrium problem then there is an infinite number of equilibrium configurations for any positive friction coefficient (in particular for arbitrary small ones). 
Under the assumptions of Proposition 7, we can denote

$$
\Phi_{\mu}=\mathbf{u}_{\mu}-\mathbf{u}_{0},
$$

and we observe that $\boldsymbol{\Phi}_{\mu}$ is a nonzero displacement field (when $0<\mu \leq \varepsilon$ ) satisfying:

$$
\left\{\begin{aligned}
\operatorname{div} \boldsymbol{\sigma}\left(\Phi_{\mu}\right) & =\mathbf{0} \quad \text { in } \Omega, \\
\boldsymbol{\sigma}\left(\boldsymbol{\Phi}_{\mu}\right) & =\mathbf{C} \boldsymbol{\varepsilon}\left(\boldsymbol{\Phi}_{\mu}\right) \quad \text { in } \Omega, \\
\boldsymbol{\Phi}_{\mu} & =\mathbf{0} \quad \text { on } \Gamma_{D}, \\
\boldsymbol{\sigma}\left(\boldsymbol{\Phi}_{\mu}\right) \mathbf{n} & =\mathbf{0} \quad \text { on } \Gamma_{N} .
\end{aligned}\right.
$$

\section{Examples of a continuum of equilibrium solutions for arbi- trary small friction}

The aim of this section is to show that there may be an infinite number of equilibrium configurations for arbitrary small $\mu>0$. More precisely we show in this section that the statement of Proposition 7 can be illustrated in the case when $\Omega$ is a triangle in $\mathbb{R}^{2}$ (whose edges represent $\Gamma_{D}, \Gamma_{N}$ and $\Gamma_{C}$ ) and the displacement field $\Phi_{\mu}=\mathbf{u}_{\mu}-\mathbf{u}_{0}$ is linear. The case of trapezoidal domains $\Omega$ leads to the same kind of results and it is also discussed. Moreover, in our example, we restrict ourselves to a class of equilibrium configurations where contact holds on $\Gamma_{C}$ so that $\left(u_{0}\right)_{n}=\left(u_{\mu}\right)_{n}=0$ on $\Gamma_{C}$. Our aim is then to construct a family of equilibrium solutions $\mathbf{u}_{\mu}$ such that $\mu \neq \bar{\mu} \Rightarrow \mathbf{u}_{\mu} \neq \mathbf{u}_{\bar{\mu}}$ for any small $\mu$ and $\bar{\mu}$.

We consider the triangle $\Omega$ of vertexes $A=(0,0), B=(1,0)$ and $C=\left(x_{c}, y_{c}\right)$ with $y_{c}>0$ and we define $\left.\Gamma_{D}=\right] B, C\left[, \Gamma_{N}=\right] A, C\left[, \Gamma_{C}=\right] A, B[$. The body $\Omega$ lies on a rigid foundation, the half-space delimited by the straight line $(A, B)$ as suggested in Figure 1.

We suppose that the body $\Omega$ is governed by Hooke's law concerning homogeneous isotropic materials so that (1.10) becomes

$$
\boldsymbol{\sigma}(\mathbf{u})=\frac{E \nu}{(1-2 \nu)(1+\nu)} \operatorname{tr}(\varepsilon(\mathbf{u})) \mathbf{I}+\frac{E}{1+\nu} \varepsilon(\mathbf{u})
$$

where I represents the identity matrix, $t r$ is the trace operator, $E$ and $\nu$ denote Young's modulus and Poisson ratio, respectively with $E>0$ and $0 \leq \nu<1 / 2$.

We begin with the determination of $\boldsymbol{\Phi}_{\mu}=\left(\left(\Phi_{\mu}\right)_{x},\left(\Phi_{\mu}\right)_{y}\right)$ in (3.1). Since $\boldsymbol{\Phi}_{\mu}$ is chosen linear and $\left(\Phi_{\mu}\right)_{y}=0$ on $\Gamma_{C} \cup \Gamma_{D}$, we get $\left(\Phi_{\mu}\right)_{y}=0$ in $\Omega$. Moreover $\left(\Phi_{\mu}\right)_{x}=0$ in $\left.\Gamma_{D}=\right] B, C[$. Hence we can search $\Phi_{\mu}$ of the following form:

$$
\begin{aligned}
& \left(\Phi_{\mu}\right)_{x}=f(\mu)\left(y_{c} x+\left(1-x_{c}\right) y-y_{c}\right) \\
& \left(\Phi_{\mu}\right)_{y}=0
\end{aligned}
$$

where $f$ is a function depending on the friction coefficient. 


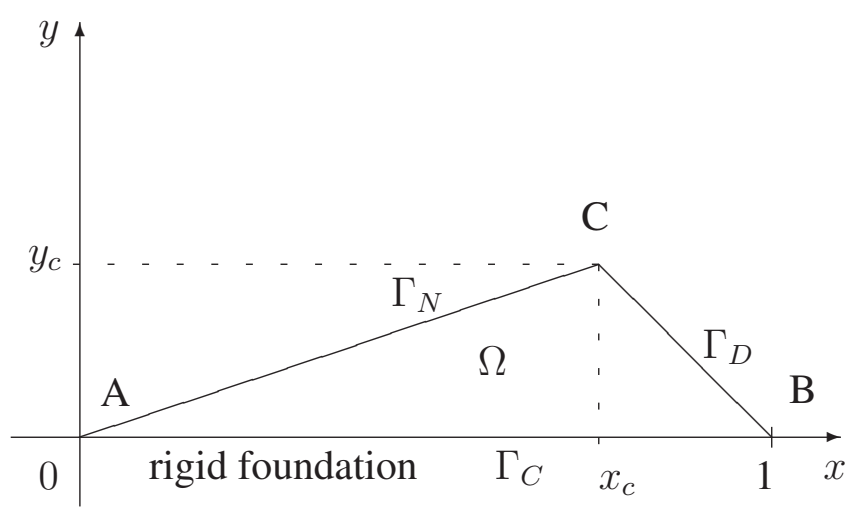

Figure 1: The geometry of the body $\Omega$

Inserting now the expressions (4.2)-(4.3) of $\Phi_{\mu}$ in the constitutive law (4.1) gives

$$
\boldsymbol{\sigma}\left(\boldsymbol{\Phi}_{\mu}\right)=\frac{f(\mu) E}{1+\nu}\left(\begin{array}{cc}
\frac{y_{c}(1-\nu)}{1-2 \nu} & \frac{1-x_{c}}{2} \\
\frac{1-x_{c}}{2} & \frac{\nu y_{c}}{1-2 \nu}
\end{array}\right),
$$

and $\operatorname{div}\left(\boldsymbol{\sigma}\left(\Phi_{\mu}\right)\right)=\mathbf{0}$. Then we consider the Neumann condition: $\boldsymbol{\sigma}\left(\Phi_{\mu}\right) \mathbf{n}=\mathbf{0}$ on $\Gamma_{N}$. Since the unit outward normal vector on $\Gamma_{N}$ is $\mathbf{n}=\left(-y_{c} / \sqrt{x_{c}^{2}+y_{c}^{2}}, x_{c} / \sqrt{x_{c}^{2}+y_{c}^{2}}\right)$, the stress vector on $\Gamma_{N}$ becomes

$$
\boldsymbol{\sigma}\left(\boldsymbol{\Phi}_{\mu}\right) \mathbf{n}=\left(\begin{array}{c}
\frac{f(\mu) E\left(2 \nu y_{c}^{2}-2 y_{c}^{2}-x_{c}^{2}+2 x_{c}^{2} \nu+x_{c}-2 x_{c} \nu\right)}{2(1-2 \nu)(1+\nu) \sqrt{x_{c}^{2}+y_{c}^{2}}} \\
\frac{f(\mu) E y_{c}\left(x_{c}-1+2 \nu\right)}{2(1-2 \nu)(1+\nu) \sqrt{x_{c}^{2}+y_{c}^{2}}}
\end{array}\right)
$$

We search for a function $f$ which does not vanish for any small positive $\mu$. Moreover we have $0 \leq \nu<1 / 2, E>0$ and $y_{c}>0$. So the Neumann condition is equivalent to the two following equalities (4.5) and (4.6):

$$
\begin{aligned}
\nu & =\frac{1-x_{c}}{2}, \\
y_{c} & =x_{c} \sqrt{\frac{1-x_{c}}{1+x_{c}}} .
\end{aligned}
$$

Hence

$$
\left.x_{c} \in\right] 0,1\left[, \quad y_{c}=x_{c} \sqrt{\frac{1-x_{c}}{1+x_{c}}} .\right.
$$


The admissible line $\gamma$ in which are located the pairs $\left(x_{c}, y_{c}\right)$ satisfying (4.7) is depicted in Figure 2 .

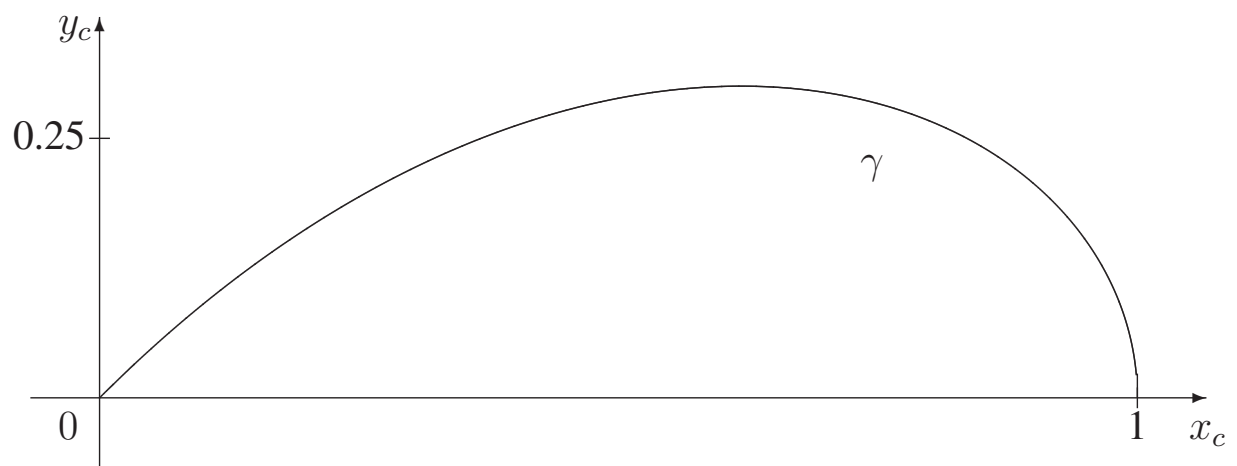

Figure 2: The admissible line $\gamma$ for point $C=\left(x_{c}, y_{c}\right)$.

In this case the normal and tangential constraints on $\Gamma_{C}$ given by (4.4), with $\mathbf{n}=(0,-1)$, $\mathbf{t}=(1,0)$, and denoting

$$
\boldsymbol{\sigma}_{T}\left(\boldsymbol{\Phi}_{\mu}\right)=\sigma_{T}\left(\boldsymbol{\Phi}_{\mu}\right) \mathbf{t}
$$

become

$$
\begin{gathered}
\sigma_{n}\left(\boldsymbol{\Phi}_{\mu}\right)=\frac{f(\mu) E\left(1-x_{c}\right)}{3-x_{c}} \sqrt{\frac{1-x_{c}}{1+x_{c}}}, \\
\sigma_{T}\left(\boldsymbol{\Phi}_{\mu}\right)=\frac{f(\mu) E\left(1-x_{c}\right)}{x_{c}-3} .
\end{gathered}
$$

Remark 8. If instead of a triangle we consider a trapezoid of vertexes $A=(0,0), B^{\prime}=(\theta, 0)$, $C=\left(x_{c}, y_{c}\right)$ and $D=\left(x_{c}+\theta\left(1-x_{c}\right),(1-\theta) y_{c}\right)$ with $y_{c}>0$ and $0<\theta<1$ the discussion is the same as previously. In fact it suffices to define $\left.\Gamma_{D}=\right] C, D\left[, \Gamma_{N}=\right] A, C[\cup] B^{\prime}, D\left[, \Gamma_{C}=\right] A, B^{\prime}[$ and to observe that the lines $A C$ and $B^{\prime} D$ are parallel (see Figure 3). The discussion is still the same in this case.

Let us now determine a field $\mathbf{u}_{0}=\left(\left(u_{0}\right)_{x},\left(u_{0}\right)_{y}\right)$ solving the frictionless equilibrium problem (1.9)-(1.14). To simplify our discussion, we search a linear displacement field $\mathbf{u}_{0}$ where $\left(u_{0}\right)_{y}=0$ on $\Gamma_{C}$ (contact holds everywhere on $\Gamma_{C}$ ). Therefore it can be written

$$
\begin{aligned}
& \left(u_{0}\right)_{x}=a x+b y+c, \\
& \left(u_{0}\right)_{y}=d y,
\end{aligned}
$$

with $a, b, c, d$ in $\mathbb{R}$. Inserting this expression in the constitutive law (4.1) implies:

$$
\boldsymbol{\sigma}\left(\mathbf{u}_{0}\right)=\frac{E}{1+\nu}\left(\begin{array}{cc}
\frac{a+\nu(d-a)}{1-2 \nu} & \frac{b}{2} \\
\frac{b}{2} & \frac{d+\nu(a-d)}{1-2 \nu}
\end{array}\right) .
$$




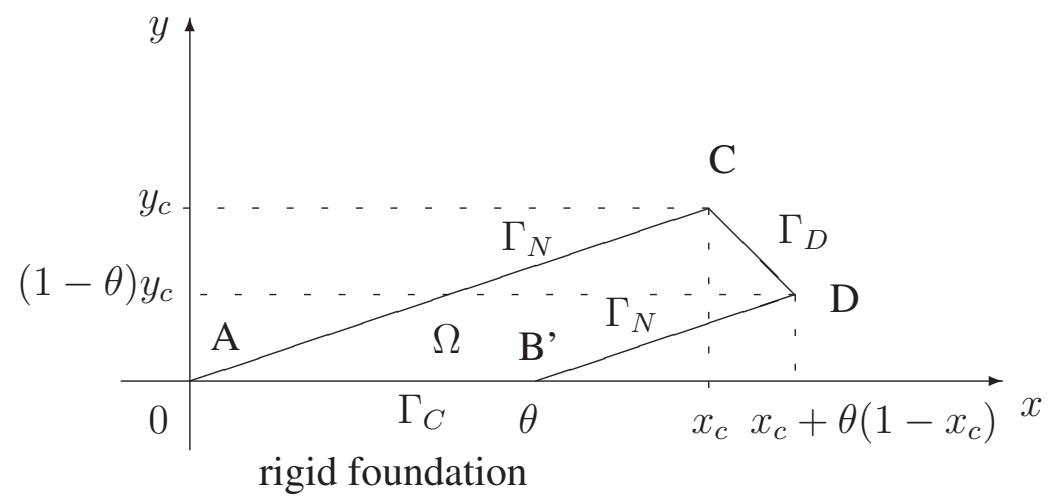

Figure 3: Case where $\Omega$ is a trapezoid

So $b=0$ since $\sigma_{T}\left(\mathbf{u}_{0}\right)=-E b /(2(1+\nu))$ on $\Gamma_{C}$. Besides, from $\nu=\left(1-x_{c}\right) / 2$ (see (4.5)), we deduce that

$$
\sigma_{n}\left(\mathbf{u}_{0}\right)=\frac{E\left(\left(1-x_{c}\right) a+\left(1+x_{c}\right) d\right)}{x_{c}\left(3-x_{c}\right)}
$$

on $\Gamma_{C}$ which requires that $\left(1-x_{c}\right) a+\left(1+x_{c}\right) d \leq 0$ according to (1.13).

The displacement field $\mathbf{U}=\left(U_{x}, U_{y}\right)$ incorporated in the Dirichlet condition (1.11) on $\Gamma_{D}$ becomes

$$
\begin{aligned}
& U_{x}=a x+c, \\
& U_{y}=d y .
\end{aligned}
$$

In the case where $\Omega$ is a triangle, the densities of surface forces $\boldsymbol{\sigma}\left(\mathbf{u}_{0}\right) \mathbf{n}=\mathbf{F}=\left(F_{x}, F_{y}\right)$ on the boundary part $\Gamma_{N}$ with $\mathbf{n}=\left(-\sqrt{\left(1-x_{c}\right) / 2}, \sqrt{\left(1+x_{c}\right) / 2}\right)$ are then

$$
\begin{aligned}
& F_{x}=-\frac{E\left(\left(1+x_{c}\right) a+\left(1-x_{c}\right) d\right) \sqrt{1-x_{c}}}{\sqrt{2} x_{c}\left(3-x_{c}\right)}, \\
& F_{y}=\frac{E\left(\left(1-x_{c}\right) a+\left(1+x_{c}\right) d\right) \sqrt{1+x_{c}}}{\sqrt{2} x_{c}\left(3-x_{c}\right)} .
\end{aligned}
$$

In the case where $\Omega$ is a trapezoid, it suffices to consider also the second part of $\Gamma_{N}$ which is precisely the straight line segment $B^{\prime} D$ depicted in Figure 3 with $\mathbf{n}=\left(\sqrt{\left(1-x_{c}\right) / 2},-\sqrt{\left(1+x_{c}\right) / 2}\right)$. Obviously $\operatorname{div}\left(\boldsymbol{\sigma}\left(\mathbf{u}_{0}\right)\right)=\mathbf{0}$, hence $\mathbf{f}=\mathbf{0}$.

Having determined a field $\mathbf{u}_{0}$ solution to the frictionless equilibrium problem (1.9)-(1.14) and a set of fields $\left(\Phi_{\mu}\right)_{\mu}$ verifying (3.2), the next step consists of adding them and to check that the 
conditions of Proposition 7 are fulfilled. From $\mathbf{u}_{\mu}=\mathbf{u}_{0}+\Phi_{\mu}$, (4.8) and (4.9) we deduce

$$
\begin{aligned}
\sigma_{n}\left(\mathbf{u}_{\mu}\right) & =\sigma_{n}\left(\mathbf{u}_{0}\right)+\sigma_{n}\left(\boldsymbol{\Phi}_{\mu}\right) \\
& =\frac{E\left(\left(1-x_{c}\right) a+\left(1+x_{c}\right) d\right)}{x_{c}\left(3-x_{c}\right)}+f(\mu) \frac{E\left(1-x_{c}\right)}{3-x_{c}} \sqrt{\frac{1-x_{c}}{1+x_{c}}} .
\end{aligned}
$$

Obviously the latter expression is nonpositive when $f(\mu) \leq 0$ which we assume next. If in addition $\sigma_{T}\left(\mathbf{u}_{\mu}\right)= \pm \mu \sigma_{n}\left(\mathbf{u}_{\mu}\right)$ then $\mathbf{u}_{\mu}$ is an equilibrium solution. The previous equality can be written as follows:

$$
\begin{aligned}
\sigma_{T}\left(\mathbf{u}_{\mu}\right) & =\sigma_{T}\left(\boldsymbol{\Phi}_{\mu}\right) \\
& =f(\mu) \frac{E\left(1-x_{c}\right)}{x_{c}-3} \\
& = \pm \mu \frac{E\left(\left(1-x_{c}\right) a+\left(1+x_{c}\right) d\right)}{x_{c}\left(3-x_{c}\right)} \pm \mu f(\mu) \frac{E\left(1-x_{c}\right)}{3-x_{c}} \sqrt{\frac{1-x_{c}}{1+x_{c}}}
\end{aligned}
$$

which implies

$$
f(\mu)=\mp \frac{\mu}{x_{c}} \frac{\left(a+\frac{1+x_{c}}{1-x_{c}} d\right)}{1 \pm \mu \sqrt{\frac{1-x_{c}}{1+x_{c}}}} .
$$

Since we assumed that $f(\mu) \leq 0$ (at least in an interval $[0, \varepsilon]$ ) and $\left(1-x_{c}\right) a+\left(1+x_{c}\right) d \leq 0$, we have necessarily $\sigma_{T}\left(\mathbf{u}_{\mu}\right)=-\mu \sigma_{n}\left(\mathbf{u}_{\mu}\right)$ and consequently

$$
f(\mu)=\frac{\mu}{x_{c}} \frac{\left(a+\frac{1+x_{c}}{1-x_{c}} d\right)}{1-\mu \sqrt{\frac{1-x_{c}}{1+x_{c}}}} \quad \text { with } \quad 0 \leq \mu<\sqrt{\frac{1+x_{c}}{1-x_{c}}} .
$$

So we obtain the expression of $\mathbf{u}_{\mu}=\left(\left(u_{\mu}\right)_{x},\left(u_{\mu}\right)_{y}\right)$ on $\Omega$ :

$$
\begin{aligned}
& \left(u_{\mu}\right)_{x}=a x+c+\mu \frac{a+\frac{1+x_{c}}{1-x_{c}} d}{1-\mu \sqrt{\frac{1-x_{c}}{1+x_{c}}}}\left(\sqrt{\frac{1-x_{c}}{1+x_{c}}}(x-1)+\frac{1-x_{c}}{x_{c}} y\right), \\
& \left(u_{\mu}\right)_{y}=d y .
\end{aligned}
$$

We then remark that the displacement field $\mathbf{u}_{\mu}$ moves points $A, B$ and $C$ to the new positions given by $\bar{A}, \bar{B}$ and $\bar{C}$ respectively.

$$
\bar{A}=\left(c+\mu \frac{a+\frac{1+x_{c}}{1-x_{c}} d}{\mu-\sqrt{\frac{1+x_{c}}{1-x_{c}}}}, 0\right)
$$




$$
\begin{gathered}
\bar{B}=(1+a+c, 0) \\
\bar{C}=\left((a+1) x_{c}+c,(1+d) x_{c} \sqrt{\frac{1-x_{c}}{1+x_{c}}}\right) .
\end{gathered}
$$

We finally have to check that $\mathbf{u}_{\mu} \neq \mathbf{u}_{\bar{\mu}}$ when $0 \leq \mu \neq \bar{\mu}<\sqrt{\left(1+x_{c}\right) /\left(1-x_{c}\right)}$ which is satisfied if we assume in addition that

$$
\left(1-x_{c}\right) a+\left(1+x_{c}\right) d<0 .
$$

In this case it is easy to check that the function $f$ defined in (4.14) is a bijection from

$$
\left[0, \sqrt{\left(1+x_{c}\right) /\left(1-x_{c}\right)}[\text { onto }]-\infty, 0\right]
$$

so that all the assumptions of Proposition 7 are satisfied.

The previous discussion and the statement of Proposition 7 prove the existence of a continuum of solutions to the equilibrium problem (1.9)-(1.14) in the cases which we describe hereafter. The result is given in the case of a triangle but the same result holds also when $\Omega$ is a trapezoid according to Remark 8.

Proposition 9. Let be given the triangle $\Omega$ of vertexes $A=(0,0), B=(1,0)$ and $C=$ $\left(x_{c}, x_{c} \sqrt{\left(1-x_{c}\right) /\left(1+x_{c}\right)}\right)$ with $\left.x_{c} \in\right] 0,1\left[\right.$. Set $\left.\Gamma_{D}=\right] B, C\left[, \quad \Gamma_{N}=\right] A, C\left[, \Gamma_{C}=\right] A, B[$. Let $E>0, \nu=\left(1-x_{c}\right) / 2$, $\mathbf{U}$ and $\mathbf{F}$ given by (4.10)-(4.11) and (4.12)-(4.13) where (4.17) holds. Assume that $\mathbf{f}=\mathbf{0}$.

If the friction coefficient $\bar{\mu}$ is such that $0<\bar{\mu}<\sqrt{\left(1+x_{c}\right) /\left(1-x_{c}\right)}$ then the problem (1.9)(1.14) admits an infinity of equilibrium configurations. More precisely any displacement field given by (4.15)-(4.16) with $0 \leq \mu \leq \bar{\mu}$ solves the problem (1.9)-(1.14).

An immediate consequence of the previous study is the following result.

Corollary 10. There exist configurations such that the equilibrium problem (1.9)-(1.14) admits a continuum of solutions for arbitrary small friction coefficients.

Finally we illustrate Proposition 9 with a simple example.

Example 11. Set $x_{c}=3 / 5, E=1, a=-1 / 2, c=-1 / 2, d=0$. According to Proposition 9, we obtain $C=(3 / 5,3 / 10), \nu=1 / 5, \mathbf{f}=\mathbf{0}$ in $\Omega, \mathbf{F}=(\sqrt{5} / 9,-\sqrt{5} / 18)$ on $\Gamma_{N}, \mathbf{U}=(-(x+1) / 2,0)$ on $\Gamma_{D}$. If the friction coefficient $\bar{\mu}$ is such that $0<\bar{\mu}<2$ then any displacement field $\mathbf{u}_{\mu}$ defined by

$$
\begin{aligned}
& \left(u_{\mu}\right)_{x}=\frac{1}{\mu-2} x+\frac{2 \mu}{3(\mu-2)} y-\frac{\mu-1}{\mu-2}, \\
& \left(u_{\mu}\right)_{y}=0
\end{aligned}
$$


with $0 \leq \mu \leq \bar{\mu}$ solves the problem (1.9)-(1.14). Figure 4 depicts the initial configuration and two deformed configurations characterized by the position of the point $\bar{A}$ (in fact the physically relevant configurations correspond to $0<\bar{\mu}<1$ ). When $\mu$ ranges from 0 to 1 , the point $\bar{A}$ moves from $(-1 / 2,0)$ to $(0,0)$. Finally we mention that this example involves important strains (although the small strain hypothesis has been adopted). Of course this is in order to have a better graphical representation and it can be avoided.

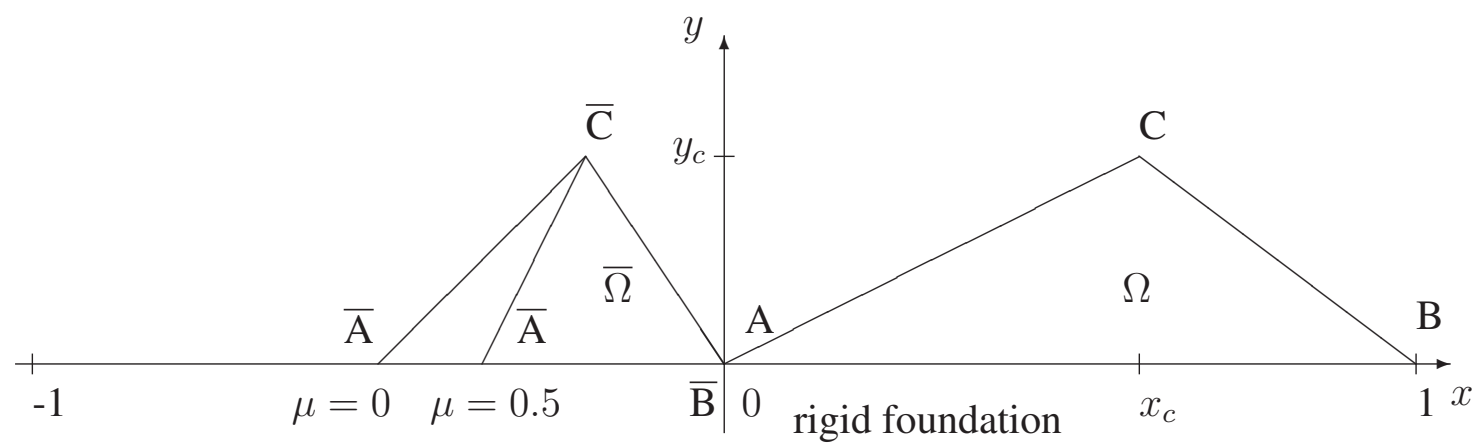

Figure 4: Initial configuration $\Omega$ and deformed configurations $\bar{\Omega}$

Remark 12. 1. An open question linked to the static problem: it is easy to check that the previous example involves equilibrium solutions which are not always solving the static friction problem. As a consequence the existence of such an example in which solution multiplicity of the static problem occurs for any positive friction coefficient is an open problem. Note that the problem remains open even with a variable geometry.

2. An open question linked to the wedged configurations: the above example involves exterior "loads" (f, $\mathbf{F}, \mathbf{U})$ which are different from $(\mathbf{0}, \mathbf{0}, \mathbf{0})$. The existence of an example in which solution multiplicity for the equilibrium problem occurs for any positive friction coefficient when $(\mathbf{f}, \mathbf{F}, \mathbf{U})=(\mathbf{0}, \mathbf{0}, \mathbf{0})$ is an open question. This would require a deeper study of wedged configurations.

\section{Perspectives: the normal compliance model}

The compliance model introduced and studied in $[13,17]$ and $[11,12]$ consists of finding the displacement field $\mathbf{u}: \Omega \times[0, T] \rightarrow \mathbb{R}^{d}$ satisfying (1.1)-(1.4), the initial conditions (1.7)-(1.8) and a contact condition of the following form:

$$
-\sigma_{n}(\mathbf{u})=c_{n}\left(u_{n}\right)_{+}^{m_{n}}
$$


where $c_{n}>0$ and $m_{n} \geq 1$ are two parameters describing the material characteristics of the contact interface and $(.)_{+}$represents the positive part. The model of friction law on $\Gamma_{C} \times[0, T]$ is now given by (with $c_{T}>0$ and $m_{T} \geq 1$ ):

$$
\left\{\begin{aligned}
\dot{\mathbf{u}}_{T}=\mathbf{0} & \Longrightarrow\left|\boldsymbol{\sigma}_{T}(\mathbf{u})\right| \leq c_{T}\left(u_{n}\right)_{+}^{m_{T}}, \\
\dot{\mathbf{u}}_{T} \neq \mathbf{0} & \Longrightarrow \boldsymbol{\sigma}_{T}(\mathbf{u})=c_{T}\left(u_{n}\right)_{+}^{m_{T}} \frac{\dot{\mathbf{u}}_{T}}{\left|\dot{\mathbf{u}}_{T}\right|} .
\end{aligned}\right.
$$

We think that the study in this paper (variational formulation of equilibrium solutions, study of multiplicities and definition of wedged configurations) could be generalized to this phenomenological law.

\section{Acknowledgements}

This work is supported by "l'Agence Nationale de la Recherche" (project JCJC018201). We are thankful to Prof. Ioan Ionescu (Université de Chambéry, France) for the fruitful discussions concerning this problem.

\section{References}

[1] L.-E. Andersson. Existence results for quasistatic contact problems with Coulomb friction, Appl. Math. Optim., 42 (2000), 169-202.

[2] J.R. Barber, P. Hild. Non-uniqueness, eigenvalue solutions and wedged configurations involving Coulomb friction, Proceedings of the IJTC 2004, ASME/STLE International Joint Tribology Conference, Long Beach California, USA, 24-27 October 2004, Part A, 127-132.

[3] C. Eck, J. Jarušek. Existence results for the static contact problem with Coulomb friction, Math. Models Meth. Appl. Sci., 8 (1998), 445-468.

[4] C. Eck, J. Jarušek, M. Krbec. Unilateral contact problems: variational methods and existence theorems, Pure and Applied Mathematics 270, CRC Press, 2005.

[5] W. Han, M. Sofonea. Quasistatic contact problems in viscoelasticity and viscoplasticity, American Mathematical Society, International Press, 2002.

[6] J. Haslinger, I. Hlaváček, J. Nečas. Numerical methods for unilateral problems in solid mechanics, in Handbook of Numerical Analysis, Volume IV, Part 2, eds. P.G. Ciarlet and J. L. Lions, North Holland, 1996, pp. 313-485.

[7] R. Hassani, I. Ionescu, E. Oudet. Critical friction for wedged configurations, Int. J. Solids Structures, 44 (2007), 6187-6200. 
[8] R. Hassani, I. Ionescu, N.-D. Sakki. Unstable perturbation of the equilibrium under Coulomb friction. Nonlinear eigenvalue analysis, Comput. Methods Appl. Mech. Engrg., 196 (2007), 2377-2389.

[9] P. Hild. Non-unique slipping in the Coulomb friction model in two-dimensional linear elasticity, Q. Jl. Mech. Appl. Math., 57 (2004), 225-235.

[10] P. Hild. Multiple solutions of stick and separation type in the Signorini model with Coulomb friction, Z. Angew. Math. Mech., 85 (2005), 673-680.

[11] A. Klarbring, A. Mikelíc, M. Shillor. Frictional contact problems with normal compliance, Int. J. Engng. Sci., 26 (1988), 811-832.

[12] A. Klarbring, A. Mikelíc, M. Shillor. On friction problems with normal compliance, Nonlinear Anal., 13 (1989), 935-955.

[13] J.A.C. Martins, J.T. Oden. Existence and uniqueness results for dynamic contact problems with nonlinear normal and friction interface laws, Nonlinear Anal., 11 (1987), 407-428.

[14] J.A.C. Martins, M.D.P. Monteiro Marques (Eds.) Contact Mechanics, Proceedings of the third Contact Mechanics International Symposium, Solid Mechanics and its Applications 103, Kluwer, 2002.

[15] C. Naéjus, A. Cimetière. Sur la formulation variationnelle du problème de Signorini avec frottement de Coulomb, C. R. Acad. Sci. Sér. I Math., 323 (1996), 307-312.

[16] J. Nečas, J. Jarušek, J. Haslinger. On the solution of the variational inequality to the Signorini problem with small friction, Bolletino U.M.I., 17 (1980), No. 5, 796-811.

[17] J.T. Oden, J.A.C. Martins. Models and computational methods for dynamic friction phenomena, Comput. Methods. Appl. Mech. Engrg., 52 (1985), 527-634.

[18] Y. Renard. A uniqueness criterion for the Signorini problem with Coulomb friction, SIAM J. Math. Anal., 38 (2006), 458-467.

[19] R. Rocca, M. Cocu. Existence and approximation of a solution to quasistatic Signorini problem with local friction, Int. J. Engrg. Sci., 39 (2001), 1233-1255.

[20] M. Shillor (Ed.) Recent advances in contact mechanics, Mathl. Comput. Modelling, 28 (1998), No. 4-8, 1-534.

[21] P. Wriggers, U. Nackenhorst (Eds.) Analysis and simulation of contact problems, Proceedings of the fourth Contact Mechanics International Symposium, Lecture Notes in Applied and Computational Mechanics 27, Springer, 2006. 